\title{
Avaliação econômica e energética da distribuição direta do etanol hidratado no estado de São Paulo
}

\author{
Mateus Brito Gama ${ }^{1}$, João Alexandre Widmer²
}

\begin{abstract}
Resumo: A presente pesquisa tem como objetivo investigar os ganhos econômicos e energéticos da distribuição direta do etanol hidratado no estado de São Paulo. Entende-se por distribuição direta a entrega da destilaria ao posto de abastecimento, sem passar compulsoriamente por uma base de distribuição de combustíveis por determinação legal. Na avaliação dos cenários foi desenvolvido um modelo de custo e outro de gasto energético. Os resultados do modelo dos custos da cadeia de distribuição mostram um ganho potencial da ordem de $47 \%$ sobre o modelo de distribuição atual. Esse valor de ganho potencial corresponde a aproximadamente $3 \%$ do valor de venda do álcool hidratado na bomba de combustível dos postos de abastecimento e, provavelmente, não tem impacto sobre a demanda do produto. Há que se considerar, também, que a viabilidade técnica da entrega direta depende da implementação de novas estruturas de armazenagem nas destilarias, o que implicaria uma redução na estimativa desse ganho potencial, num período inicial de amortização de investimentos. Por outro lado, o ganho estimado em reais é da ordem de R \$ 260 milhões/ano, o que, uma vez amortizados os investimentos em armazenagem, representa um valor anual significativo se a alternativa de distribuição direta fosse implementada e repassada para o consumidor final do produto. Com relação ao gasto energético, os resultados apresentam uma estimativa de ganho da ordem de 470 mi de MJ por ano, um ganho de $28 \%$ sobre o consumo atual. Comparando os valores de gasto energético encontrados nesta pesquisa com outros valores divulgados na literatura para a mesma etapa da cadeia, observa-se que, de um lado não existe um consenso entre os autores sobre a ordem de grandeza deste valor, e de outro, o valor do gasto energético da cadeia de distribuição é relativamente pequeno quando comparado com o consumo energético dos outros estágios da cadeia de produção da cana-de-açúcar. Por outro lado, o ganho anual corresponde à ordem de grandeza de emissão de gases de efeito estufa de uma cidade de pequeno porte com aproximadamente 40 mil habitantes.
\end{abstract}

Palavras-chave: distribuição, etanol hidratado, economia de transportes, SIG, custo, energia.

Abstract: This study aims to investigate the economic and energetic gains of direct distribution of hydrous ethanol in the state of São Paulo. Direct distribution means the direct delivery of the product from the distillery to the gas station, without the compulsory passage through a distribution base due to a legal statement. A cost and an energy consumption model to compare the scenarios were developed. The model results for the distribution chain costs show a potential gain of $47 \%$ over the present distribution model. This value corresponds to a potential gain of approximately $3 \%$ of the sales value of hydrous ethanol at the gas stations and probably has no influence over the product demand. It is also important to consider that direct delivery depends on the implementation of new storage structures at the distilleries, which would imply a reduction in the estimate of this potential gain during an initial period of investment amortization. The estimated annual gain of R\$ 260 million, however, would represent a significant value if transferred to the end consumers once the investment in tankage is paid for. With respect to energy consumption, the results show a potential gain of 470 million MJ per year, with a gain of $28 \%$ over the present distribution model. Comparing the energy consumption values obtained in this research, with other values reported in the literature, one observes that there is not a consensus about the magnitude of this value among the authors, and also that the energy consumption in the distribution chain is relatively small when compared with the other stages of the sugar cane production chain. On the other side, the annual gain corresponds to the order of magnitude of a small city of approximately 40.000 inhabitants.

Keywords: distribution, hydrous ethanol, transportation economy, GIS, cost, energy.

\section{INTRODUÇÃO}

\subsection{O contexto da pesquisa}

Atualmente, o etanol brasileiro proveniente da cana de açúcar já é consolidado como uma alternativa à gasolina, principalmente depois da inserção da tecnologia Flex Fuel, a partir de 2003, na qual é possível utilizar tanto o etanol hidratado, como o etanol anidro, misturado com a gasolina. Mais que isso, há uma potencialização do uso dessa nova

\footnotetext{
1 Mateus Brito Gama, Departamento de Engenharia de Transportes,

EESC-USP. (mateus.gama@uol.com.br)

2 João Alexandre Widmer, Departamento de Engenharia de Transportes,

EESC-USP. (widmer@sc.usp.br)

Manuscrito recebido em 26/08/2015 e aprovado para publicação em 08/09/2015.

Este artigo é parte de TRANSPORTES v. 23, n. 4, 2015. ISSN: 2237-1346
}

(online). DOI: 10.14295/transportes.v23i4.990 alternativa, principalmente pelas questões ambientais, no que se refere à redução da emissão de gases poluentes e a finitude da matéria prima dos combustíveis fósseis (CAMELINI e CASTILLO, 2012).

Contudo, para expandir o consumo de etanol no Brasil e no mundo, em substituição a outros combustíveis fósseis, não basta a justificativa que a fonte é renovável, ou que a tecnologia para sua utilização já está dominada. O produto tem que ser viável para produtores e consumidores. Neste aspecto, é relevante avaliar tanto a viabilidade econômica como a energética dos processos existentes na cadeia de produção do etanol, para identificar não só o custo envolvido no processamento do etanol ao longo dessa cadeia, mas também o consumo de energia proveniente de combustíveis fósseis para produzir e transportar o referido combustível desde o processo de plantio da cana até a entrega do produto ao consumidor final. 


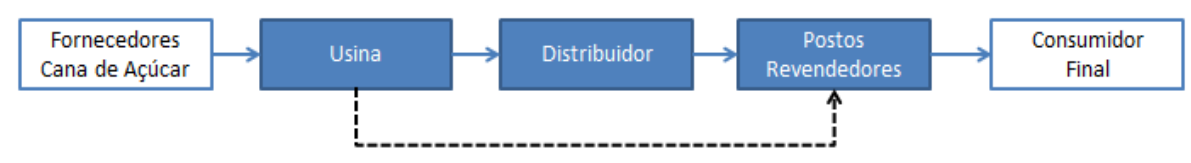

\section{$\longrightarrow$ Fluxo Real ------> Fluxo Estudado}

Figura 1. Representação dos fluxos real e estudado na cadeia de suprimento do etanol

O objetivo deste trabalho é apresentar os resultados de um modelo de distribuição direta do produto desde a destilaria de origem ao posto de abastecimento do consumidor final, sem passar pelas bases regionais de distribuição de combustíveis, frente ao cenário atual de passagem compulsória pelas bases regionais de distribuição de combustíveis.

A rota compulsória via base regional de combustíveis é estabelecida pela agência reguladora de combustíveis no Brasil, a Agência Nacional do Petróleo (ANP). Com base na resolução $\mathrm{N}^{\circ} 43$ da ANP, a usina só pode comercializar o etanol, hidratado ou anidro, com o distribuidor, ficando vedada a entrega direta aos postos revendedores.

Essa resolução, tem por finalidade concentrar o controle de qualidade e a arrecadação de tributos em poucos agentes, neste caso as distribuidoras de combustíveis, o que facilita a ação do órgão regulador. Sob os aspectos econômicos e energéticos da cadeia, entretanto, constitui o problema do "turismo do álcool" (YOSHISAKI, MUSCAT e BIAZZI, 1996), que afeta, por hipótese, as regiões produtoras de etanol que estão próximas das regiões de consumo, gerando a movimentação do etanol até um distribuidor para posteriormente voltar para os locais de consumo. Essa operação configura, em princípio, um gasto econômico e energético desnecessário, que poderia ser minimizado se fosse implantado um ambiente operacional com nota fiscal eletrônica, emitida pelos postos revendedores, associada à entrega do etanol direta das usinas até os postos de combustíveis, dado que a auditoria do órgão regulador na ponta da cadeia é determinante para assegurar a qualidade do produto fornecido ao consumidor final.

Além dos aspectos legais e dos interesses comerciais envolvidos na interação produtores/distribuidores, a questão de concentrar o controle nos distribuidores também pode ser explicada pela eventual limitação da ANP de efetivamente gerenciar o processo e auditar a qualidade do produto ao longo de toda a cadeia de distribuição. Levantamentos recentes listam 341 usinas produtoras, das quais 159 estão localizadas no estado de São Paulo; 225 bases de distribuição, 47 em São Paulo; e 38.148 postos de revenda (BRASIL, 2013b; ANP, 2013; MENEZES, RODRIGUES e VAZQUEZ, 2013).

Os órgãos de classe que representam as destilarias de etanol entendem que essa intermediação dos distribuidores no escoamento da produção gera custos desnecessários de transporte e argumentam que se as usinas já produzem, estocam e analisam a qualidade do produto, poderiam entregá-lo diretamente aos postos de abastecimento nas regiões de consumo próximas às unidades produtoras. A passagem pelas distribuidoras se justifica apenas no caso do etanol anidro, dado o reprocessamento para misturá-lo à gasolina (LOPES, SILVA e CONEJERO, 2010).

Em termos de modelagem do problema econômico do fluxo de distribuição do produto é possível adotar um modelo baseado nos custos operacionais do transporte ou, alternativamente, nos preços praticados no mercado de fretes, dado que na rede estudada do Estado de São Paulo, o modo de transporte rodoviário é, praticamente, o único modo de transporte utilizado (BNDES e CGEE, 2008).

Assim, no contexto desta pesquisa adotou-se o frete rodoviário como variável próximo para a estimativa do custo de distribuição (SIFRECA - USP/ESALQ-LOG).

A Figura 1 ilustra, sinteticamente, a cadeia logística da produção e distribuição do etanol e permite identificar o foco da investigação apresentada neste trabalho.

A melhor representação prática do problema envolve a entrega direta do etanol hidratado no estado de São Paulo, onde há o maior número de centros produtores de etanol do país. Assim, a avaliação quantitativa concentrou-se nos ganhos econômicos e energéticos do cenário de distribuição direta do etanol hidratado nesse Estado. De maneira complementar os ganhos energéticos constituirão mais um valor de referência e poderão ser comparados com os valores divulgados na literatura para esse estágio da cadeia logística.

\subsection{Consumo de combustíveis fósseis na produção de biocombustíveis}

Nos últimos anos, muito se tem discutido sobre a situação e as perspectivas de consumo de fontes de energia não renovável, tanto no âmbito nacional, como internacional. Estas discussões recaem, principalmente, na questão da energia fóssil proveniente do carvão mineral e do petróleo, devido à vasta aplicabilidade desses componentes minerais. Nesse contexto, um dos setores que mais se destaca é o de transportes, pelo fato de depender fortemente de fontes de energia de origem fóssil para funcionar.

No ano de 2012, nos Estados Unidos (EUA), 95,4\% da energia consumida pelo setor de transportes era proveniente de combustíveis fósseis (EIA, 2013). O setor, neste mesmo país, representou 27,2\% da emissão de gases efeito estufa, no ano de 2011 (EPA, 2013). No Brasil, em 2012, $87,5 \%$ da energia consumida pelo setor eram de fonte não renovável, e o mesmo representou $48,8 \%$ da emissão de gases efeito estufa no país (BRASIL, 2013a).

A relevância do setor de transportes no consumo de combustíveis fósseis tem reflexos diretos nos impactos ambientais. Diante disso, umas das soluções para mitigar esses impactos é a utilização de fontes renováveis de energia. Destaca-se a energia derivada de biomassa que produz o biodiesel e o etanol. Na matriz energética brasileira, segundo BRASIL (2013a), a parcela de energia de fonte renovável já representa $42,4 \%$, com destaque para a biomassa proveniente da cana de açúcar, que responde por $15,4 \%$ da matriz total, tanto na produção de eletricidade (bagaço da cana), como de etanol.

O etanol (ou álcool etílico) é um biocombustível que pode ser obtido através do processamento de várias culturas agrícolas, destacando-se a produção a partir do milho, com 
Tabela 1. Gastos energéticos na produção e distribuição do etanol (GJ/ha)

\begin{tabular}{llllll}
\hline $\begin{array}{l}\text { Inputs de energia } \\
\text { por estágio (GJ/ha) }\end{array}$ & $\begin{array}{l}\text { Oliveira et al. } \\
\text { (2005) }\end{array}$ & $\begin{array}{l}\text { Pimentel } \boldsymbol{P} \\
\text { Patzek } \\
\text { (2008) }\end{array}$ & $\begin{array}{l}\text { Macedo, Seabra e } \\
\text { Silva (2008) }\end{array}$ & $\begin{array}{l}\text { Boddey et al. } \\
\text { (2008) }\end{array}$ & $\begin{array}{l}\text { Andreoli, Pimentel e } \\
\text { Souza (2012) }\end{array}$ \\
\hline Agrícola (A) & 35,98 & 25,36 & 16,19 & 12,71 & 30,22 \\
Industrial & 3,63 & 18,62 & 1,82 & 2,61 & 0,69 \\
Distribuição (D) & 2,82 & 7,62 & $-{ }^{-}$ & & 3,09 \\
D/A & $8 \%$ & $30 \%$ & & 15,32 & $10 \%$ \\
Total & 42,43 & 51,6 & 18,01 & 34 \\
\hline
\end{tabular}

${ }^{\mathrm{a}}$ Distribuição não foi considerada no trabalho.

maior expressão nos Estados Unidos, e da cana de açúcar no Brasil (GOLDEMBERG, COELHO e GUARDABASSI, 2008). No caso da cana de açúcar, seu processamento tem como principais produtos o etanol anidro, utilizado em mistura com a gasolina; o etanol hidratado, utilizado puro em veículos automotores; e o açúcar. Além desses, são gerados subprodutos, dos quais se destaca a energia elétrica gerada a partir da queima do bagaço da cana.

No que tange à viabilidade energética dos biocombustíveis, entretanto, diversos estudos vêm sendo realizados no sentido de comparar a energia fóssil investida na produção (inputs), com aquela produzida (output). Apesar dos estudos possuírem um procedimento de contabilização semelhante, observa-se grande divergência nos resultados dos gastos energéticos associados ao processo de produção e distribuição do etanol, em especial aquele proveniente da cana de açúcar no Brasil, como mostram os dados apresentados na Tabela 1 para três estágios da cadeia logística do etanol: agrícola, industrial e distribuição. Nota-se a dispersão dos resultados encontrados pelos vários autores quanto à ordem de grandeza do consumo energético em cada estágio da cadeia de produção. Essa diferença está relacionada a diversos fatores, que incluem as estimativas de consumo de insumos e materiais, os coeficientes energéticos adotados e a contabilização, ou não, da utilização da energia elétrica produzida pelo bagaço da cana na etapa industrial.

Outro ponto observado nesses estudos é o peso das atividades agrícolas e a ênfase dos pesquisadores nas atividades agrícola e industrial, sendo que alguns não incluem a distribuição do produto. Entretanto, mesmo com menor impacto nos consumos totais, quando comparado à etapa agrícola (Tabela 1-linha D/A), há um gasto de energia de origem fóssil no transporte do etanol das usinas até os postos de combustíveis a ser contabilizado.

\section{MÉTODO}

O método proposto na presente pesquisa é o da construção de cenários, auxiliado por uma ferramenta de SIG (Sistema de Informação Geográfica). Para facilitar a compreensão do método as etapas que foram desenvolvidas no trabalho são descritas a seguir.

\subsection{Construção de uma rede de transportes e coleta de bases de dados georreferenciados}

O principal objetivo dessa etapa foi obter uma base consistente de dados que permitisse montar a matriz das distâncias entre todos os nós da rede de transportes. Para isso foram coletadas bases de dados georreferenciados com as seguintes informações: malha rodoviária do estado; localização das usinas produtoras de etanol; localização das bases de distribuição; e localização dos municípios.

\subsection{Obtenção dos dados de produção, transbordo e consumo.}

Nesta etapa foram levantados os dados de localização e quantitativos referentes à produção de etanol nos nós de origem, transbordo nas bases de distribuição e consumo de etanol nos nós de destino (municípios do estado de São Paulo). Adotou-se a hipótese que o centro geográfico das cidades representa o baricentro do consumo de álcool hidratado de cada município e que o consumo é proporcional à população. Para modelar o cenário de produção e consumo de etanol, os dados foram ajustados a uma mesma escala temporal, tendo sido adotada a hipótese de que todo o álcool produzido é consumido no mesmo período anual. Adotaram-se valores de produção e de consumo no período de um ano, utilizando-se a produção de etanol referente à safra 2011/2012 e o consumo do ano de 2012.

\subsection{Elaboração de um modelo de custo e gasto energético por unidade de distância, e escolha do modelo matemático de minimização.}

Outra etapa do trabalho, além da construção da rede, refere-se à elaboração dos modelos que representam os custos e os gastos energéticos na distribuição do etanol. A representação dos custos da distribuição do etanol hidratado no estado de São Paulo foi feita em um modelo de frete com variação linear em função da distância percorrida. Já o modelo de gastos energéticos na distribuição do etanol baseouse no consumo de combustível fóssil (diesel) dos caminhões tanque utilizados na distribuição. Adotou-se um modelo linear de consumo de combustível $(1 / \mathrm{km})$ pela distância percorrida na rede.

A representação dos custos da distribuição do etanol hidratado no estado de São Paulo foi baseada em um modelo do frete rodoviário por distância percorrida e custos unitários de transbordo e armazenagem:

- Transporte do Produto $\left(\mathrm{T}_{\mathrm{p}}\right)$ : refere-se à movimentação do produto.

- Operação de Tancagem $\left(\mathrm{O}_{\mathrm{t}}\right)$ : referente ao transbordo e armazenagem.

Para modelar o primeiro componente foram obtidos valores de fretes cobrados para o transporte de etanol, tendo como origem e destino cidades do estado de São Paulo. Esses dados de frete rodoviário foram coletados no Sistema de Informações de Fretes (SIFRECA), desenvolvido pelo Grupo de Pesquisa e Extensão em Logística Agroindustrial (ESALQ-LOG) para o produto álcool (anidro e hidratado). No total, foram coletadas 21 tarifas $\left(\mathrm{R} \$ / \mathrm{m}^{3}\right)$, relacionadas 
com a distância $(\mathrm{km})$ dos trechos correspondentes a cada uma delas. A partir da plotagem dos valores no software MS Excel foi proposto um modelo de regressão linear como hipótese básica de modelagem.

Este modelo de regressão linear foi adotado visando à simplificação da relação entre as duas variáveis, já que para uma variação relativamente pequena de distância, há uma tendência de crescimento proporcional da tarifa conforme sugerido em Ballou (2010) ("Tarifas Proporcionais").

Desta forma, o modelo de custo de transportte é representado pela expressão (1):

$$
T_{p}=(0,17 d+9,26) q
$$

Em que

$d$ : distância $(\mathrm{km})$ entre o nó de origem e o nó de destino;

$q$ : quantidade $\left(\mathrm{m}^{3}\right)$ de etanol alocada para cada origem e destino.

Nos resultados deste estudo não foram incorporadas informações específicas de pedágios e os custos a eles associados, dado que se admitiu que os dados de frete publicados no SIFRECA incorporam, ainda que de uma forma imprecisa, esse componente de custo do transporte, e que os resultados do modelo são apresentados de uma forma agregada de fluxo em todo o sistema rodoviário do Estado.

O segundo componente de custo refere-se ao transbordo e armazenagem. Para o contexto pesquisado, estes podem ser sintetizados em uma única operação que será denominada de 'operação de tancagem'. Esta representa a descarga do caminhão que transporta etanol nas bases de distribuição (transbordo), utilização da tangem (armazenagem) e carga do caminhão (transbordo) que transporta etanol para os postos de abastecimento.

De acordo com as Tarifas de Referência para Serviços de Movimentação de Etanol, divulgada pela TRANSPETRO, o custo para realizar a operação de tancagem em um terminal localizado em Guarulhos, no estado de São Paulo, é de R \$ 21,62 por $\mathrm{m}^{3}$. Este valor é cobrado para períodos de 15 dias de armazenagem, sendo cobrado o mesmo valor caso seja ultrapassado este tempo (TRANSPETRO, 2013).

Diante disso, foi adotado este valor no modelo como uma impedância, ou seja, quando o etanol passar pela base, foi contabilizado um gasto por quantidade de produto a ser descarregado/carregado e armazenado, expresso em $\mathrm{R} \$ / \mathrm{m}^{3}$. tancagem:

A expressão (2) representa o modelo da operação de

$$
O_{t}=(21,62) Q
$$

Onde $Q$ : quantidade $\left(\mathrm{m}^{3}\right)$ de etanol processado nas bases de distribuição.

Baseado nos dois componentes apresentados, a modelagem do Custo Total ( $C_{T}$ ) na distribuição será feita como mostra a expressão 3:

$$
C_{T}=T_{p}+O_{t}
$$

Já o modelo de gastos energéticos na distribuição do etanol baseou-se no consumo de combustível fóssil (diesel) dos caminhões tanque utilizados na distribuição. Adotou-se um modelo linear de consumo de combustível $(1 / \mathrm{km})$ pela distância percorrida na rede.
Baseado na quantidade de combustível consumido, foi feita a conversão de unidades de volume para unidades energéticas considerando-se o valor calorífico do diesel. A expressão (4) representa os gastos energéticos totais da distribuição:

$$
E_{T}=C_{c} \cdot d \cdot v_{c} \cdot n
$$

Em que,

$E_{T}$ : gastos energéticos totais (MJ);

$C_{c}$ : consumo de combustível do caminhão tanque $(1 / \mathrm{km})$;

$d$ : distância $(\mathrm{km})$ entre o nó de origem e o nó de destino;

$v_{c}:$ valor calorífico do diesel (MJ/1);

\subsection{Ferramenta de análise dos custos e consumo energético na rede de transporte.}

Como ferramenta de geração de soluções utilizou-se o software TransCAD ${ }^{\circledR}$, um SIG voltado especificamente para problemas de transporte, o qual contém um pacote de ferramentas voltadas para análise e otimização de fluxos em redes. As variáveis independentes estabelecidas para os problemas se resumem em distância e quantidade transportada. Para encontrar a menor distância entre as origens e destinos (ODs) adotou-se o modelo referente ao problema do fluxo de mínimo custo. Já para a determinação da quantidade alocada em cada arco da rede foram aplicados os modelos do problema do transporte e do problema de transbordo em uma rede não capacitada, buscando alocar o recurso (etanol produzido) com um custo mínimo (distância) entre os nós de oferta e os nós de demanda.

\subsection{Geração dos cenários}

Com base na rede de transportes e nos modelos apresentados anteriormente foram avaliados o custo e o gasto energético em dois cenários. O Cenário 1 se refere ao cenário atual de distribuição do etanol hidratado no estado de São Paulo, em que o produto sai das usinas, passa pelas bases de distribuição, para então ser levado para aos municípios consumidores (Problema do Transbordo). O Cenário 2 representa a entrega direta do etanol hidratado no Estado, no qual este será transportado diretamente das usinas para os municípios (Problema do Transporte).

\section{RESULTADOS}

A partir da simulação dos fluxos na rede de acordo com as premissas do Cenário 1 e do Cenário 2, foram calculados os valores de custo $(\mathrm{R} \$)$ e gasto energético $(\mathrm{MJ})$ referentes à atividade de transporte do produto na cadeia de distribuição usina - consumidor final. Na sequência apresenta-se, para cada cenário, uma tabela com os resultados intermediários que compõe os valores totais de custo e gasto energético.

Cenário 1 - Distribuição via bases regionais

A Tabela 2 apresenta os resultados obtidos com a simulação do Cenário 1 .

Como pode ser observado, os valores foram separados por etapa da cadeia, sendo a primeira referente à distribuição do etanol entre as usinas e as bases de distribuição, e a segunda entre as bases e os municípios. Ao final das 
Tabela 2. Resultados obtidos para o Cenário 1

\begin{tabular}{|c|c|c|c|}
\hline \multicolumn{4}{|c|}{ CENÁRIO 1} \\
\hline USINA - BASE & & & un. \\
\hline PRODUÇÃO ALOCADA NA BASE & & $5.834 .459,55$ & $m^{3}$ \\
\hline DISTÂNCIA MÉDIA PONDERADA & & 268,35 & $\mathrm{~km}$ \\
\hline \multicolumn{4}{|l|}{ CUSTO U-B } \\
\hline & TRANSPORTE & $310.557 .500,33$ & $R \$$ \\
\hline & TRANSBORDO & $126.141 .015,44$ & $R \$$ \\
\hline & SUBTOTAL & $436.698 .515,77$ & $R \$$ \\
\hline \multicolumn{4}{|l|}{ ENERGIA U-B } \\
\hline & TRANSPORTE & $1.343 .703 .360,37$ & $M J$ \\
\hline \multicolumn{4}{|l|}{ BASE - MUNICÍPIOS } \\
\hline PRODUÇÃO ALOCADA NOS MUNICÍPIOS & & $5.834 .459,55$ & $m^{3}$ \\
\hline DISTÂNCIA MÉDIA PONDERADA & & 68,93 & $\mathrm{~km}$ \\
\hline \multicolumn{4}{|l|}{ CUSTO B-M } \\
\hline & TRANSPORTE & $122.393 .290,41$ & $R \$$ \\
\hline \multicolumn{4}{|l|}{ ENERGIA B-M } \\
\hline \multicolumn{4}{|l|}{ RESUMO } \\
\hline CUSTO TOTAL $\left(\mathrm{C}_{\mathrm{T}}\right)$ & & $559.091 .806,18$ & $R \$$ \\
\hline ENERGIA TOTAL $\left(\mathrm{E}_{\mathrm{T}}\right)$ & & $1.688 .056 .688,44$ & $M J$ \\
\hline \multicolumn{4}{|c|}{ Tabela 3. Resultados obtidos para o Cenário 2} \\
\hline \multicolumn{4}{|c|}{ CENÁRIO 2 } \\
\hline USINA - MUNICÍPIOS & & & un. \\
\hline PRODUÇÃO ALOCADA NOS MUNICÍPIOS & & $5.834 .459,55$ & $m^{3}$ \\
\hline DISTÂNCIA MÉDIA PERCORRIDA & & 243,58 & $\mathrm{~km}$ \\
\hline CUSTO TOTAL $\left(\mathrm{C}_{\mathrm{T}}\right)$ & & 295.625.561,09 & $R \$$ \\
\hline ENERGIA TOTAL $\left(\mathrm{E}_{\mathrm{T}}\right)$ & & 1.218.666.046,17 & $M J$ \\
\hline
\end{tabular}

duas é apresentado um resumo com a soma do custo total CT e da energia total - ET. O custo resultante deste cenário para o ano em estudo foi de $\mathrm{R} \$ 559,1$ milhões, sendo composto da seguinte maneira: $55 \%(\mathrm{R} \$ 310,6$ milhões) pelo custo de transporte Usina-Base; $23 \%$ ( $\mathrm{R} \$ 126,1$ milhões) pelo custo de transbordo; $22 \%$ ( $\mathrm{R} \$ 122,4$ milhões) pelo custo de transporte Base-Município.

No que tange o gasto energético, este resultou em um montante de 1,7 bilhões de MJ, sendo $80 \%$ (1,34 bilhões de MJ) referente ao transporte Usina-Base, e 20\% (0,34 bilhões de MJ) ao transporte Base-Município. Cabe ressaltar que o modelo de gasto energético adotado na pesquisa não contemplou a operação de tancagem nas bases de distribuição, admitindo-se que os bombeamentos são feitos com motores elétricos alimentados por energia proveniente de hidrelétricas.

Cenário 2 - Distribuição direta indústria posto de abastecimento

Os resultados da simulação do Cenário 2 estão dispostos na Tabela 3. O custo total do transporte de etanol hidratado a partir da entrega direta foi de $R \$ 295,6$ milhões, enquanto que o gasto energético foi de 1,2 bilhões de $M J$. A distância média percorrida entre as usinas e municípios foi $243,6 \mathrm{~km}$.

\subsection{Fluxos do etanol hidratado no estado de São Paulo}

Os resultados apresentados mostram que as distâncias médias percorridas nos deslocamentos Usina-Base (Cenário 1) e Usina-Município (Cenário 2) são semelhantes, sendo a do primeiro 268,4 km, e a do segundo 243,6 $\mathrm{km}$. Ao mesmo tempo, a distância média encontrada no deslocamento Base-Município (Cenário 1) apresenta um valor TRANSPORTES v. 23, n. 4 (2015), p. 13-20 bem menor $(68,9 \mathrm{~km})$. Tal fato está relacionado ao distanciamento que existe entre as regiões de maior produção de etanol hidratado no estado, com as regiões de maior consumo (municípios) e as regiões com maior transbordo (bases).

A partir da análise do consumo de etanol hidratado no estado, observa-se que os principais municípios consumidores estão localizados na região sudeste do Estado. Dentre esses municípios se destaca a cidade de São Paulo, responsável por aproximadamente $24 \%$ do consumo de etanol hidratado do estado. De modo semelhante ao consumo, o transbordo também se concentra nessa região, sendo a cidade de Paulínia e a cidade de São Paulo responsáveis por aproximadamente $43 \%$ da capacidade de armazenagem do estado.

Diferente do que foi observado no consumo e no transbordo, a concentração da produção de etanol no estado encontra-se nas regiões oeste e noroeste do estado. Essa diferença de concentração entre a oferta (produção das usinas) e demanda (consumo dos municípios e transbordo das bases), acaba provocando deslocamentos médios relativamente grandes no transporte do produto entre as origens e destinos. A Figura 2 ilustra essa questão.

Outro aspecto investigado nesta pesquisa foi o possível impacto do fluxo de etanol sobre o sistema rodoviário nos links da rede simulada. A partir das tabelas de fluxo fornecidas pelo TransCAD nas aplicações do Problema de Transportes, foi gerado o fluxo equivalente de veículos tipo em trechos de maior carregamento da rede de transportes e comparou-se esse valor com o fluxo estimado a partir do Volume Médio Diário de Tráfego (VDM) de veículos comerciais em trechos específicos de rodovias do estado (SÃO PAULO, 2012). A partir dessa comparação, pode-se 

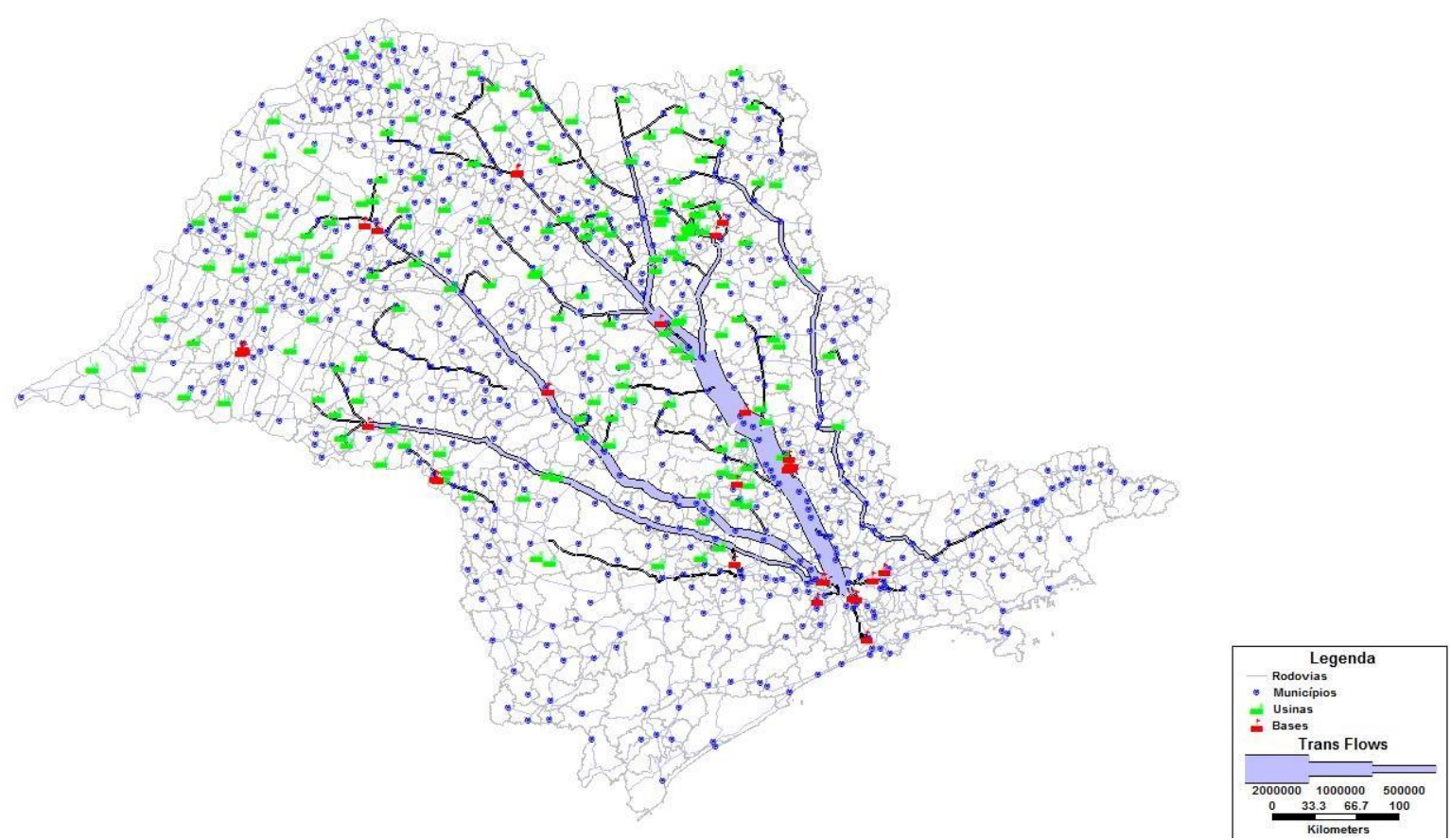

Figura 2. Mapa com a representação do fluxo do produto nos links entre as usinas e os municípios, gerado pelo TransCAD

Tabela 4. Comparacão dos custos e gastos energéticos dos Cenários 1 e 2

\begin{tabular}{|c|c|c|c|}
\hline & CENÁRIO 1 & CENÁRIO 2 & $R E D U C \tilde{A} O$ \\
\hline CUSTO (R\$) & $559.091 .806,18$ & $295.625 .561,09$ & $47 \%$ \\
\hline $\begin{array}{l}\text { GASTO ENERGÉTICO } \\
(\mathrm{MJ})\end{array}$ & $1.688 .056 .688,44$ & 1.218.666.046,17 & $28 \%$ \\
\hline
\end{tabular}

afirmar que os valores encontrados na pesquisa não têm, em termos de VDM, efeito significativo sobre o nível de serviço do sistema viário utilizado.

\subsection{Avaliação comparativa dos cenários}

A partir dos resultados apresentados, foi estabelecido um comparativo entre os cenários para avaliar qual o ganho obtido com a entrega direta do etanol hidratado (Cenário 2), em termos de custo e energia, com relação à entrega praticada atualmente (Cenário 1). Como pode ser observado na Tabela 4 houve uma redução de $47 \%$ no custo total, com um ganho econômico estimado de $\mathrm{R} \$ 263,46$ milhões. No que tange ao gasto energético houve uma redução de $28 \%$, para uma estimativa de ganho de 469,39 mi de MJ.

Do ganho obtido em termos de custo, $52 \%$ referemse ao transporte do etanol hidratado e $48 \%$ ao transbordo, o que significa que houve uma redução real de $25 \%$ no custo de transporte e de $23 \%$ no custo da operação de processo nas bases, a qual inclui a descarga do produto que vem das usinas, o custo de armazenagem e teste de qualidade e a recarga de caminhões para a distribuição aos postos de abastecimento.

Se for considerado que na entrega direta do etanol seria necessária a implementação de novas estruturas de armazenagem próximas às usinas, para suprir a ausência de armazenagem nas bases, como descreve a literatura, podese considerar que parte da economia obtida no custo de transbordo poderia ser convertida investimentos em áreas de tancagem nas indústrias.

Adotando o preço médio do etanol hidratado ao consumidor no estado de São Paulo de R \$ 1,806 por litro, no ano de 2012 (ANP, 2013a), e estabelecendo a relação do 18 primeiro ganho econômico obtido na pesquisa $(\mathrm{R} \$ 263,5$ milhões) com o total transportado (5,8 milhões de $\left.\mathrm{m}^{3}\right)$, obtém-se uma economia de aproximadamente $\mathrm{R} \$ 0,05$ por litro. Este valor representa cerca de $3 \%$ do preço final do produto, o que não parece ser tão representativo ao ponto de torná-lo mais competitivo em relação à gasolina, que é o combustível que compete diretamente com o etanol.

$\mathrm{O}$ valor do ganho energético anual, percentualmente menor que o do ganho econômico, não permite uma avaliação objetiva dos tomadores de decisão envolvidos no processo (Ministério do Meio Ambiente, ANP, distribuidoras de combustíveis e produtores de etanol), mas uma conversão desse valor em emissão de gases de efeito estufa, especificamente $\mathrm{o} \mathrm{CO}_{2}$, ilustra melhor a dimensão desse resultado.

$\mathrm{Na}$ conversão utilizou-se um fator de emissão de $\mathrm{CO}_{2}$ para veículos pesados a diesel de 74 g/MJ (ÁLVARES JUNIOR E LINKE, 2001), o que resultou, quando relacionado ao ganho energético (469,4 milhões de MJ), em 34,7 mil t de $\mathrm{CO}_{2}$, que deixariam de ser emitidos em um ano. Este valor corresponde à ordem de grandeza de emissão de gases de efeito estufa de uma cidade de pequeno porte com aproximadamente 40 mil habitantes como, por exemplo, Socorro no interior do estado de São Paulo (SÃO PAULO, 2013).

\subsection{Análise comparativa do gasto energético com a literatura}

Outro objetivo da pesquisa foi comparar o gasto energético obtido com valores divulgados na literatura para a etapa de distribuição da cadeia do etanol. Para realizar a 
Tabela 5. Gastos energéticos da etapa de distribuição encontrados na literatura e na pesquisa

\begin{tabular}{lc}
\hline Trabalhos da literatura & $\begin{array}{c}\text { Gasto energético na distri- } \\
\text { buição do etanol (GJ/ha) }\end{array}$ \\
\hline Oliveira et al. (2005) & 2,82 \\
Pimentel e Patzek (2008) & 7,62 \\
Andreoli, Pimentel e Souza & 3,09 \\
(2012) & \\
\hline \multicolumn{2}{c}{ Pesquisa } \\
Cenário 1 \\
Cenário 2 \\
\hline
\end{tabular}

comparação foram utilizados os dados da Tabela 2, apresentada na revisão bibliográfica deste trabalho, na qual constam os gastos energéticos de três trabalhos expressos em GJ/ha.

Para padronizar as unidades, os gastos energéticos encontrados para os cenários de avaliação desta pesquisa foram convertidos incialmente para $\mathrm{MJ} / \mathrm{l}$, estabelecendo a relação entre $\mathrm{E}_{\mathrm{T}}$ e o total transportado, e depois para $\mathrm{GJ} / \mathrm{ha}$, adotando uma produtividade de 6314 1/ha (TRIANA, 2011). A partir desse procedimento, o gasto energético encontrado para o Cenário 1 foi de $1,83 \mathrm{GJ} / \mathrm{ha}$, e para o Cenário 2 foi de 1,32 GJ/ha, como mostra a Tabela 5, ambos menores que os valores encontrados nas referências bibliográficas citadas neste estudo.

Essa divergência nos valores encontrados na pesquisa, com aqueles divulgados na literatura, especialmente com o trabalho de Pimentel e Patzek (2008) pode ser creditada ao ceticismo desses dois autores quanto ao etanol proveniente da cana no Brasil, o que, talvez, tenha impactado negativamente a contabilização do gasto energético. Não foi possível identificar a origem das divergências entre os dados da pesquisa com os outros trabalhos, pois os autores desses artigos não explicitam os parâmetros utilizados no cálculo.

Cabe destacar ainda que, mesmo sabendo da importância da etapa de distribuição na cadeia, fica evidente que a mesma agrega relativamente pouco ao consumo energético, quando comparada com as atividades agrícolas da cadeia de produção. Comparando-se o gasto obtido no Cenário $1,1,83 \mathrm{GJ} / \mathrm{ha}$, com o gasto relativo à atividade agrícola divulgado no trabalho de Andreoli, Pimentel e Souza (2012), 30,22 GJ/ha, percebe-se que o primeiro representa apenas $6 \%$ do segundo.

\section{CONCLUSÕES}

Esta pesquisa teve por objetivo investigar os ganhos econômicos e energéticos da distribuição direta do etanol hidratado no estado de São Paulo. Para isso foram criados dois cenários de avaliação, o primeiro, representando o sistema atual de distribuição, em que o etanol sai das destilarias, realiza o transbordo nas bases, para então chegar aos municípios de consumo; e o segundo, representando a entrega direta das destilarias até os municípios.

Em termos de ganhos econômicos houve uma redução estimada de $47 \%$ no custo total, com um ganho econômico da ordem de $\mathrm{R} \$ 263$ milhões por ano. A viabilização da entrega direta, entretanto, depende da implementação de novas estruturas de armazenagem nas usinas, bem como de um novo sistema de controle de qualidade e arrecadação de tributos, o que implicaria a necessidade de investimentos.
Assim, o ganho real, numa primeira fase, devido principalmente aos investimentos nas capacidades de armazenagem nas indústrias, estaria limitado ao ganho no custo de transportes, que representa cerca de $\mathrm{R} \$ 137$ milhões por ano, ou seja, da ordem de metade da estimativa do ganho anual.

Conclui-se, também, que o ganho real tem pequena participação no preço final do produto, e que o cenário do processo de distribuição não parece significativo para influenciar a demanda do produto pelo consumidor final. Isso não significa que deva ser desconsiderado, pois afinal, por conta do atual sistema de distribuição, a sociedade (consumidor final) acaba pagando mais caro por uma rede de distribuição ineficiente.

Esta ineficiência pode estar associada não só às restrições legais (regulamentação), mas também às questões estratégicas das empresas que dominam o mercado de distribuição. As corporações que atuam nesse mercado, utilizam o sistema atual de distribuição do etanol como forma de otimizar os processos de maximização do seu desempenho corporativo.

Com relação ao gasto energético, se estimou um ganho de $28 \%$ sobre o consumo energético do cenário atual. O ganho da ordem de 470 milhões de MJ por ano, apesar de pequeno quando comparado com o consumo energético de toda a cadeia produtiva do etanol, corresponde à emissão de gases de efeito estufa de uma cidade com cerca de 40.000 habitantes.

Comparando-se o ganho energético por área plantada de cana-de-açúcar com outros valores divulgados na literatura para o mesmo estágio da cadeia, os valores estimados neste estudo são significativamente menores que os das outras referências bibliográficas.

\section{REFERÊNCIAS}

Álvares Junior, O. M.; Linke, R. R. A. (2001). Metodologia Simplificada de Cálculo das Emissões de Gases do Efeito Estufa de Frotas de Veículos no Brasil. CETESB. São Paulo, 2001.

Andreoli, C.; Pimentel, D.; Souza, S. P. (2012). Net energy balance and carbono footprint of biofuel from corn and sugarcane. In: Pimentel, D. (ed.). Global economic and environmental aspects of biofuels. CRC Press, Boca Raton. 221248

ANP (2013). Relação de bases de distribuição de combustíveis líquidos autorizadas a operar: planilha eletrônica - atualizado em setembro de 2013. Disponível em: www.anp.gov.br. Acesso em: (05/09/2013).

ANP (2013a). Anuário estatístico brasileiro do petróleo gás natural e biocombustíveis. Rio de Janeiro. 
Banco Nacional de Desenvolvimento Econômico e Social (2008). Bioetanol de cana-de-açucar: energia para o desenvolvimento sustentável. Rio de Janeiro. 312 p.

BRASIL. Ministério das Minas e Energia (2013a). Balanço energético nacional 2013: ano base 2012 - relatório síntese. Brasília, DF; Rio de Janeiro: EPE. 55 p.

BRASIL. Ministério da Agricultura, Pecuária e Abastecimento (2013b). Relação das unidades produtoras cadastradas no Departamento da Cana-de-açucar e Agronergia. Disponível em: www.agricultura.gov.br/arq_editor/file/Desenvolvimento_Susten tavel/Agroenergia/Orientacoes

Tecnicas/Usinas\%20e\%20Destilarias\%20Cadastradas/DADOS_ PRODUTOES_20_03_2012.pdf. Acesso em: (12/08/2013).

Boddey, R. M.; Soares, L. H. B.; Alves, B. J. R., Urquiaga, S. (2008). Bio-ethanol production in Brazil. In: Pimentel, D. (ed.). Biofuels, solar and wind as renewable energy systems. Springer, New York. p. 331-356.

CALIPER (2006a). TransCAD transportation GIS software: user's guide. Caliper Corporation, Newton. 804 p.

Camelini, J. H.; Castillo, R. (2012). Logística e competitividade no circuito espacial produtivo do etanol no Brasil. Boletim Campineiro de Geografia, v. 2, n. 2, p. 262-278.

EIA (2013). Monthly energy review. Washington, DC. 201 p. il. Disponível em: www.eia.gov/ totalenergy/data/ monthly/pdf/mer.pdf. Acesso em (12/08/2013).

EPA (2013). Draft inventory of US greenhouse gas emissions and sinks: 1990-2011. Washington, DC. 470 p.

Goldemberg, J.; Coelho, S. T.; Guardabassi, P. (2008). The sustainability of ethanol production from sugarcane. Energy Policy, v. 36, n. 6, p. 2086-2097.

DOI:10.1016/j.enpol.2008.02.028

LMC International (2008). Ethanol Quarterly: Q3. Disponível em: www.lmc.co.uk/Biofuels_and_Biomass-

LMC_Ethanol_Market_Report. Acesso em: (27/02/2013).

Lopes, M. B.; Silva, A. L.; Conejero, M. A. (2010). Fluxos e poder nos canais de distribuição de etanol carburante: um estudo qualitativo no Estado de São Paulo. Revista de Administração, v. 45 , n. 4 , p. 356-372.

Macedo, I. C.; Seabra, J. E. A.; Silva, J. E. A. R. (2008). Green house gases emissions in the production and use of ethanol from sugarcane in Brazil: the 2005/2006 averages and a prediction for 2020. Biomass and Bioenergy, v. 32, n. 7, p. 582-595.

DOI:10.1016/j.biombioe.2007.12.006

Menezes, R. C.; Rodrigues, T. S.; Vazquez, M. G. C. (2013). Implementação da Solução de Integração da Cadeia de Suprimentos de Etanol. Anais do 17 SAP Forum Brasil, SAP, São Paulo.

Oliveira, M. E. D.; Vaughan, B. E.; Rykiel, E. J. (2005). Ethanol as fuel: energy, carbon dioxide balances, and ecological footprint. BioScience, v. 55, n. 7, p. 593-602.

DOI:org/10.1641/0006-3568(2005)055 [0593:

EAFECD]2.0.CO;2

Pimentel, D.; Patzek, T. W. (2008). Ethanol production: energy and economic issues related to US and Brazilian sugarcane. In:
Pimentel, D. (ed.). Biofuels, solar and wind as renewable energy systems. Springer, Dordrecht. cap. 14, p. 357-371.

SÃO PAULO (2012). Estatística de tráfego: volume médio diário (VDM). Departamento de Estradas e Rodagem. Secretaria de Logística e Transportes, São Paulo. Disponível em: http://200.144.30.103/vdm/SFCG_Concessionaria.asp?CodRodo via $=$. Acesso em: (10/09/2013).

SÃO PAULO (2013). Anuário Estatístico de Energéticos por Município no Estado de São Paulo - 2012. Secretaria de Energia , São Paulo.

SIFRECA (2013) Mercado de fretes. Disponível em: http://esalqlog.esalq.usp.br/sifreca/. Acesso em: (10/09/2013).

Triana, C. A. R. (2011). Energetics of Brazilian etanol: Comparison between assessment approaches. Energy Policy, Surrey, v. 39, n. 8, p. 4605-4613.

DOI:10.1016/j.enpol.2011.05.001

UNICA (2013). UNICAData. Disponível em: www.unicadata.com.br /histórico-de-produção-emoagem.php?idMn=32\&tipoHistorico=4. Acesso em: (12/02/2013).

Yoshizaki, H. T. Y.; Muscat, A. R. N.; Biazzi, J. L. (1996) Decentralizing ethanol Distribution in Southeastern Brazil. Interfaces, Catonsville, v. 26, n. 6, p. 24-34. DOI.org/10.1287/inte.26.6.24 